\title{
Diamagnetic Susceptibility of a Confined Donor in a Quantum Dot with Different Confinements
}

\author{
A. J. Peter ${ }^{1}$ and J. Ebenezar ${ }^{2}$ \\ ${ }^{1}$ Government Arts College, Melur-625 106, India \\ ${ }^{2}$ Jamal Mohamed College, Trichy-620 020, India \\ Received 21 September, accepted in final revised form 5 April 2009
}

\begin{abstract}
The binding energies of shallow hydrogenic impurity in a GaAs/GaAlAs quantum dot with spherical confinement, harmonic oscillator-like and rectangular well-like potentials are calculated as a function of dot radius using a variational procedure within the effective mass approximation. The calculations of the binding energy of the donor impurity as a function of the system geometry have been investigated. A comparison of the eigenstates of a hydrogenic impurity in all the confinements of dots is discussed in detail. We have computed and compared the susceptibility for a hydrogenic donor in a spherical confinement, harmonic oscillator-like and rectangular well-like potentials for a finite QD and observe a strong influence of the shape of confining potential and geometry of the dot on the susceptibility.
\end{abstract}

Keywords: Quantum dot; Quantum well wire; Quantum well; Diamagnetic susceptibility; Donor impurity.

(C) 2009 JSR Publications. ISSN: 2070-0237 (Print); 2070-0245 (Online). All rights reserved.

DOI: $10.3329 /$ jsr.v1i2.1184

\section{Introduction}

Semiconductor quantum dots (QDs) unique features make them promising candidates for novel semiconductor devices [1]. This is because the study of the impurity states in these low dimensional structures is an important aspect on which many theoretical and experimental works are based. The deep understanding of the effects of impurities on the electronic states of semiconductor heterostructures is a fundamental issue in semiconductor physics because their presence dramatically alter the performance of quantum devices and tier optical and transport properties [2]. The binding energy of shallow donor impurities in nanoscopic systems depends on materials and geometry, size and shape, although it seems that shape has minor influence $[3,4]$.

\footnotetext{
${ }^{1}$ Corresponding author: a.john.peter@gmail.com
} 
Using the variational method, Porras-Montenegro and Perez-Merchancano [5] studied a hydrogenic impurity in spherical QD systems with both in finite and finite barriers. The results reveal that, as the size of the QD decreases for an infinite barrier, the binding energy of the impurity increases monotonically, and for a finite barrier, the binding energy increases to their maxima and then sharply decreases. The effect of parabolic confinement on the binding energy of shallow hydrogenic impurities in a spherical quantum dot has been computed as a function of the dot dimension for different impurity positions and also as a function of the impurity position for different dot sizes [6]. They show that the impurity binding energy increases with the reduction in the dot dimension. Also the binding energy is found to depend on the location of the impurity, and the same is the maximum for the on-centre impurity.

In addition, quantum dots are excellent testing grounds for the applicability of various theoretical models. The effect of confinement can be seen either by varying the barrier height or by varying the dot size. Since in semiconductor applications dopants are used it is also of interest to see how a hydrogenic donor will behave under confined geometries. Several investigators have treated this problem either variationally with infinite barriers [7] or with spherical confinement [8]. A harmonic oscillator like potential has been used for confinement in few other investigations [9]. The binding energies and density of states of shallow impurities in spherical $\mathrm{GaAs} / \mathrm{Ga}_{1-\mathrm{x}} \mathrm{Al}_{\mathrm{x}} \mathrm{As}$ quantum dots have been calculated as a function of radius and the position of the impurity in the dot [10]. The more real zero dimensional quantum heterostructure (cubic dot) was studied by Ribeiro and Latge [3]. They found that the values of donor binding energies for cubic and spherical quantum dots are very close, provided the dots have similar volumes.

The pressure dependence of diamagnetic susceptibility of a donor has been worked out recently [11]. Diamagnetic susceptibility of shallow donors in $\mathrm{Si}$ is computed incorporating the multivalley conduction band structure and the spheroidal energy surfaces [12]. The diamagnetic susceptibility of a hydrogenic donor impurity has also been computed in low dimensional semiconductor systems (LDSS) in the infinite barrier model recently [13]. The effect of dimensionality on the binding energy of a donor has thus been shown both in the parabolic and non-parabolic band models in an low dimensional semiconductor system. One can also observe quantum chaos through diamagnetic susceptibility and electronic conductivity of electron gas in these nano structures.

In this work, calculations of binding energies of the donor impurities in GaAs quantum dot with the barrier of AlGaAs, placed at the centre are performed using the effective mass approximation within a variational scheme. The dependence of the binding energy of a shallow hydrogenic of a spherical QD is evaluated with the QD sizes. A systematic study of variation of binding energy as the function of dot size has been attempted with parabolic confinement, spherical confinement and a cubical confinement with a rectangular potential well of a quantum dot. A comparison of the eigenstates of a hydrogenic impurity in all the confinements of dots is discussed in detail. We present the shift of diamagnetic susceptibility of a hydrogenic donor impurity in $\mathrm{GaAs} / \mathrm{Ga}_{1-\mathrm{x}} \mathrm{Al}_{\mathrm{x}} \mathrm{As}$ 
quantum dot systems with the different confinements. To our knowledge, there are no theoretical or experimental reports on these aspects. The method followed is presented in Section 2 while the results and discussion are provided in Section 3.

\section{Theory and Model}

The Hamiltonian of the hydrogenic donor impurity, in the effective mass approximation, in a $\mathrm{GaAs} / \mathrm{Ga}_{1-\mathrm{x}} \mathrm{Al}_{\mathrm{x}} \mathrm{As}$ quantum dot, in the influence of electric field is given by

$$
H=-\frac{\hbar^{2}}{2 m^{*}} \nabla^{2}-\frac{e^{2}}{\varepsilon(r) r}+V_{D}
$$

where $m^{*}$ is the electronic effective mass, $\varepsilon(\bar{r})$ the dielectric function of the QD material, and $V_{D}=\frac{V r^{2}}{R^{2}}$ for $|r| \leq R$ and $V$ for $|r|>R$ where $V$ is the barrier that binds

the carrier in the dot, whose value depends upon the $\mathrm{Al}$ concentration $(x)$ in $\mathrm{Ga}_{1-\mathrm{x}} \mathrm{Al}_{\mathrm{x}} \mathrm{As}$ which is the barrier medium in which we have assumed to have embedded the GaAs dot. Using for the band gap difference, $E_{g}=1.555 x+0.37 x^{2}(\mathrm{eV})$ and assuming $60 \%$ contribution to the conduction band, for $x=0.2$, the value of $\mathrm{V}$ turns out to be $147.4 \mathrm{meV}$.

The units of length and energy used throughout are the effective Bohr radius $R^{*}=\hbar^{2} \varepsilon_{o} / m^{*} e^{2}$ and the effective Rydberg $R_{y}^{*}=m * e^{4} / 2 \varepsilon_{o}^{2} \hbar^{2}$ where $\varepsilon_{o}$ is the dielectric constant and $m^{*}$ is the effective mass of electron in the conduction band minimum of GaAs.

In these units, the Hamiltonian given in Eq. (1) becomes,

$$
H=-\nabla^{2}-\frac{2}{r}+\frac{V_{D}}{R_{y}^{*}}
$$

$V_{D}$ is the parabolic confinement.

\subsection{Finite parabolic dot}

The ground state energy of an electron in a parabolic quantum dot is with electric fields is estimated by variational method. We have assumed the trial wave function

$$
\begin{array}{ll}
\psi_{\text {in }}(r)=N_{2} \frac{\sin \left(k_{1} r\right)}{r} e^{-\xi r^{2}} & r \leq R \\
\psi_{\text {out }}(r)=N_{3} e^{-k_{2} r} e^{-\xi r^{2}} & r>R
\end{array}
$$


where $\mathrm{N}_{2}, \mathrm{~N}_{3}$ are normalization constants. By matching the wave functions and their derivatives at the boundaries of the QD, along with the normalization, we fix the values of $\mathrm{N}_{2}, \mathrm{~N}_{3}$ and $k_{1}=\left(2 m * E / \hbar^{2}\right)^{1 / 2}$ and $k_{2}=\left[2 m *(E-V) / \hbar^{2}\right]^{1 / 2}$. We take $\xi$ as the variational parameter. Since the inclusion of impurity potential leads to a nonseparable differential equation which cannot be solved analytically it is necessary to use a variational approach to calculate the eigen function and eigen value of the Hamiltonian for the ground state. Taking into account the parabolic confining geometry and the hydrogenic impurity potential, we use a trial wave function for the ground state with the impurity present is taken as

$$
\begin{array}{ll}
\psi_{\text {in }}(r)=N_{4} \frac{\sin \left(k_{1} r\right)}{r} e^{-\xi r^{2}} e^{-\alpha_{1} r} & r \leq R \\
\psi_{\text {out }}(r)=N_{5} e^{-\xi r^{2}} e^{-k_{2} r} e^{-\alpha_{1} r} & r>R
\end{array}
$$

where $\alpha_{1}$ is are the variational parameter and $\mathrm{N}_{4}, \mathrm{~N}_{5}$ are normalization constants. The second term in the wave function Eq. (6) refers the Gaussian type model and the third term describes the wave function of the impurity of a donor. It is obvious that the trail wave function has two variational parameters.

\subsection{Finite spherical dot}

The ground state energy of an electron in a spherical quantum dot is with electric fields is estimated by variational method. We have assumed the trial wave function

$$
\begin{array}{ll}
\psi_{\text {in }}(r)=N_{6} \frac{\sin \left(k_{1} r\right)}{r} & r \leq R \\
\psi_{\text {out }}(r)=N_{7} e^{-k_{2} r} & r>R
\end{array}
$$

where $\mathrm{N}_{6}, \quad \mathrm{~N}_{7}$ are normalization constants, $k_{1}=\left(2 m^{*} E / \hbar^{2}\right)^{1 / 2}$ and $k_{2}=\left[2 m^{*}(E-V) / \hbar^{2}\right]^{1 / 2}$. By matching the wave functions and their derivatives at the boundaries of the QD, along with the normalization, we fix the values of $\mathrm{N}_{6}$ and $\mathrm{N}_{7}$, taking $v_{1}$ as variational parameter and the value of $V$ is taken to be $147.4 \mathrm{meV}$.

We use a trial wave function for the ground state with the impurity present is taken as

$$
\begin{array}{ll}
\psi_{\text {in }}(r)=N_{8} \frac{\sin \left(k_{1} r\right)}{r} e^{-\alpha_{3} r} & r \leq R \\
\psi_{\text {out }}(r)=N_{9} e^{-k_{2} r} e^{-\alpha_{3} r} & r>R
\end{array}
$$

where $\alpha_{3}$ is the variational parameter and $\mathrm{N}_{8}, \mathrm{~N}_{9}$ are normalization constants. 


\subsection{Finite cubical dot}

The ground state energy of an electron in a spherical quantum dot is with electric fields is estimated by variational method. We have assumed the trial wave function

$$
\begin{aligned}
& \psi_{\text {in }}(r)=N_{10} e^{-k_{3}\left(x^{2}+y^{2}+z^{2}\right)^{1 / 2}} \cos (\pi x / L) \cos (\pi y / L) \cos (\pi z / L) \quad|x|,|y| \text { and }|z|<L / 2 \\
& \psi_{\text {out }(r)}=N_{11} e^{-k_{4}\left(x^{2}+y^{2}+z^{2}\right)^{1 / 2}} \quad|x|,|y| \text { and }|z|>L / 2
\end{aligned}
$$

where $\mathrm{N}_{10}, \quad \mathrm{~N}_{11}$ are normalization constants, $k_{3}=\left(2 m^{*} E / \hbar^{2}\right)^{1 / 2} \quad$ and $k_{4}=\left[2 m *(E-V) / \hbar^{2}\right]^{1 / 2}$. By matching the wave functions and their derivatives at the boundaries of the QD, along with the normalization, we fix the values of $\mathrm{N}_{10}$ and $\mathrm{N}_{11}$, and we take $v_{2}$ as variational parameter and the value of $V$ is taken to be $147.4 \mathrm{meV}$.

We use a trial wave function for the ground state with the impurity present is taken as

$$
\begin{aligned}
& \psi_{\text {in }}(r)=N_{12} e^{-k_{3}\left(x^{2}+y^{2}+z^{2}\right)^{1 / 2}} \cos (\pi x / L) \cos (\pi y / L) \cos (\pi z / L) e^{-\alpha_{5}\left(x^{2}+y^{2}+z^{2}\right)^{1 / 2}} \quad|x|,|y| \text { and }|z|<L / 2 \\
& \psi_{\text {out }(r)}=N_{13} e^{-k_{4}\left(x^{2}+y^{2}+z^{2}\right)^{1 / 2}} e^{-\alpha_{5}\left(x^{2}+y^{2}+z^{2}\right)^{1 / 2}} \quad|x|,|y| \text { and }|z|>L / 2
\end{aligned}
$$

where $\alpha_{5}$ is the variational parameter and $\mathrm{N}_{12}, \mathrm{~N}_{13}$ are normalization constants.

The ionization energy is given by

$$
E_{\text {ion }}=E_{\text {sub }}-\langle H\rangle_{\min }
$$

Thus the ionization energy is obtained, varying $\alpha_{5}$ for different dot sizes.

\subsection{Diamagnetic susceptibility}

The Schrödinger equation is solved variationally to find the ground state wave function which has been used in the computation of diamagnetic susceptibility $\left(\chi_{\text {dia }}\right)$ of the hydrogenic donor in QD given as [14]

$$
\chi_{\text {dia }}=-\frac{e^{2}}{6 m^{*} \varepsilon_{o} c^{2}}\left\langle r^{2}\right\rangle
$$

where $c$ is the velocity of light and is the mean square distance of the electrons from the nucleus.

\section{Results and Discussion}

Our results are presented in reduced atomic units which correspond to a length unit of an effective Bohr radius $R^{*}=\hbar^{2} \varepsilon_{o} / m * e^{2}$ and an energy unit of an effective Rydberg 
$R_{y}^{*}=m^{*} e^{4} / 2 \varepsilon_{o}^{2} \hbar^{2}$ where $\varepsilon_{o}$ is the dielectric constant and $m *$ is the effective mass of an electron in the conduction band minimum of GaAs. For GaAs/ GaAlAs quantum dots, these units are $R^{*}=103 A^{o}$ and $R^{*}=5.29 \mathrm{meV}$. In our calculations, we have assumed a spherical effective mass for the donors and included the variations in the effective mass and dielectric constants throughout QD heterostructures.

We assume that the band gap discontinuity on a GaAs/ $\mathrm{Ga}_{1-\mathrm{x}} \mathrm{Al}_{\mathrm{x}} \mathrm{As}$ QD heterostructure is distributed about $20 \%$ on the valence band and $60 \%$ on the conduction band with the total band gap difference $\Delta E_{g}$ between $\mathrm{GaAs}$ and $\mathrm{Ga}_{1-\mathrm{x}} \mathrm{Al}_{\mathrm{x}} \mathrm{As}$ given as a function of the Al concentration $x=0.2$ and $E_{g}=1.555 x+0.37 x^{2}$. For a finite barrier case we choose $x=0.2$; hence $V=36.97$ Ry*.

The variation of lowest binding energy with dot sizes in a finite dot for three different confinements is shown in Fig. 1. In all the cases, the energy increases as the radius decreases and the energy goes to zero in the bulk limit for large radii. The decrease in energy with the increase of dot size is a common feature [15].

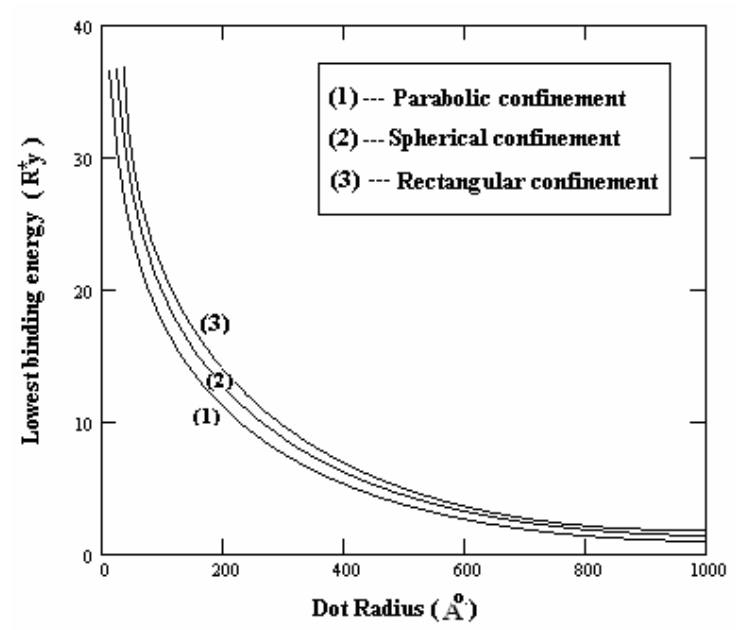

Fig. 1. Variation of lowest binding energy with dot sizes for (1) parabolic, (2) spherical, and (3) rectangular confinements.

In Fig. 2 we present the ionization energy as a function of the dot radius for a hydrogenic donor for three different confinements. As the dot radius decreases the binding energy increases, reaches a maximum and then diminishes to a limiting value corresponding to a particular radius of the dot. It is observed that the binding energy goes to $1 R^{*}$ in the bulk limit for the larger radius. The value of binding energies is more when the rectangular confinement is included in the potential. The binding energy is more when the rectangular confinement is used compared with the other geometries. This indicates that all the potentials are alike representations of the quantum-dot barrier 
potential for a radius $R$. comparing the donor binding energies for cubic, spherical and parabolic quantum dots, we found that the values are very close provided the dots are smaller radii. Ribeiro and Latgè [3] have found the values of binding energies, for cubic and spherical quantum dots are very close provided the dots have similar volumes, which are good agreement with our results.

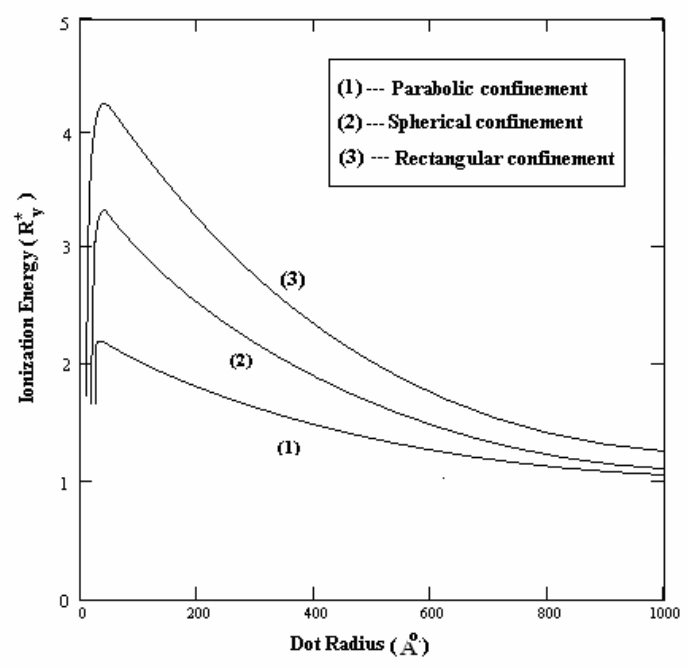

Fig. 2. Variation of ionization energy with dot sizes for (1) parabolic, (2) spherical, and (3) rectangular confinements.

In Fig. 3, we compare the susceptibility for a hydrogenic donor in all the confinements for various $R$ and the results are presented. We notice that the susceptibility value is higher for the donor confined in cubical QD with rectangular potential well than for a donor confined in a parabolic and spherical QD, since the wave function is highly localized and hence $\left\langle r^{2}\right\rangle$ is higher in the cubical dot. This indicates that there is a strong influence of the shape of the confining potential and geometry of the dot on susceptibility. The veracity of our susceptibility value is justified by considering the bulk limit value as $R \rightarrow \infty,\left\langle r^{2}>\rightarrow 3 R^{*}\left(R^{*}\right.\right.$, effective Bohr radius) and hence $\chi_{\text {dia }} \rightarrow-1.1$ a.u $\left(-2.36 \times 10^{-6}\right.$ $\mathrm{cm}^{3} /$ mole).

In the present work, the variational approaches are used in a thorough study of the binding energy of on-center shallow donors in spherical GaAs $/ \mathrm{Ga}_{1-\mathrm{x}} \mathrm{Al}_{\mathrm{x}} \mathrm{As}$ quantum dots with potential barriers taken for $r>R$ parabolic isotropic barriers and rectangular confinements. The binding energy has been calculated following a variational procedure within the effective mass approximation. We have compared the rectangular confinement with other confinements such as spherical and parabolic potentials as done in Ref. [16] in which the effect of diamagnetic susceptibility is not included. We have computed and compared the susceptibility for a hydrogenic donor in a spherical confinement, harmonic 
oscillator-like and rectangular well-like potentials for a finite QD and observe a strong influence of the shape of confining potential and geometry of the dot on the susceptibility.

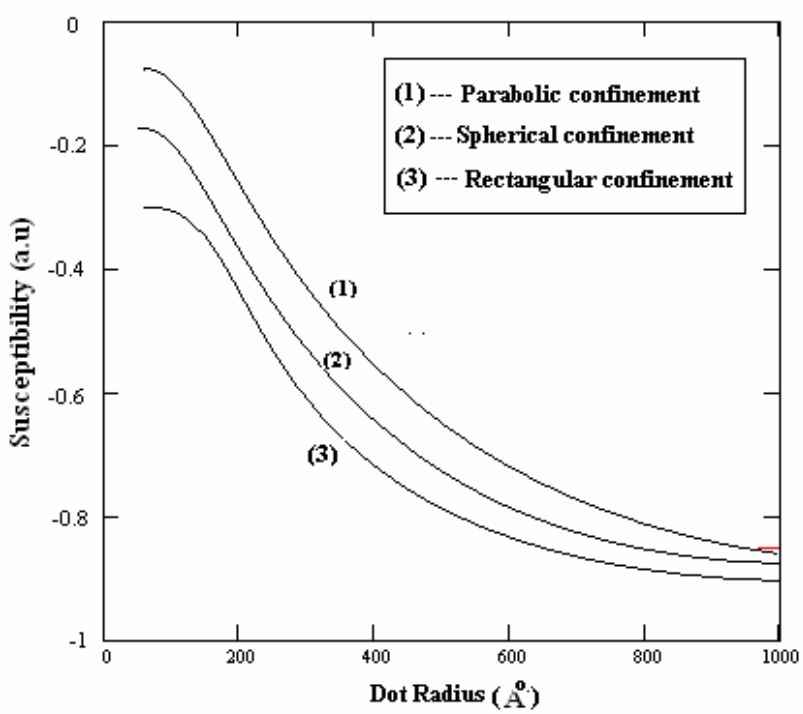

Fig. 3. Comparison of susceptibility with dot sizes for (1) parabolic, (2) spherical, and (3) rectangular confinements.

We believe that our investigations on the susceptibility of a donor in a quantum dot will be relevant to the interpretation of semiconductor-metal transition [17] in LDSS. The emerging field of spintronics also requires an extensive study of magnetic properties of nano systems and also facilitates an understanding of quantum chaos and electronic conductivity of electron gas in the nano structure. Further results are required for large values of $\mathrm{Al}$ concentration, $x$ and for narrow dots to test the effective mass theory. Experimental efforts are encouraged to lend support to our calculations and the susceptibility will soon be made to throw more light on donor wave function in confined systems.

\section{Acknowledgements}

We would like to acknowledge Ms.Babiana for her computational work on this work.

\section{References}

1. V. Shchukin, N. N. Ledentsov, and D. Bimberg, Epitaxy of Nanostructures, Nanoscience and Technology (Springer, New York, 2003).

2. H. J. Queisser and E. E. Haller, Science 281, 945 (1998). doi:10.1126/science.281.5379.945 
3. F. J. Ribeiro and A. Latge, Phys. Rev. B 50, 4913 (1994). doi:10.1103/PhysRevB.50.4913

4. P. G. Bolcatto and C. R. Proetto, Phys. Rev. B 59, 12487 (1999). doi:10.1103/PhysRevB.59.12487

5. N. Porras-Montenegro and S. T. Perez-Merchancano, Phys. Rev. B 46, 9780 (1992). doi:10.1103/PhysRevB.46.9780

6. C. Bose, J. Appl. Phys. 83, 3089 (1998). doi:10.1063/1.367065

7. J. J. Vivas-Moreno and W. Porras-Montenegro, Phys. Status. Solidi (b) 210, 723 (1998). doi:10.1002/(SICI)1521-3951(199812)210:2<723::AID-PSSB723>3.0.CO;2-T

8. N. Porras Montenegro, S. T. Pèrez-Merchancano, and A. Latge, J.Appl.Phys. 74, 7624 (1993). doi: $10.1063 / 1.354943$

9. H. O. Oyoko, C. A. Duque, N. Porras-Montenegro, J. Appl. Phy. 90, 819 (2001). doi:10.1063/1.1372976

10. W. Porras-Montenegro, S.T.Pérez-Merchancano and A. Latgé, J. Appl. Phys.74, 7652 (1993). doi:10.1063/1.354943

11. A. Elangovan and K. Navaneethakrishnan, Phys. Rev. B 48, 7986 (1993). doi:10.1103/PhysRevB.48.7986

12. Vudan Rao Hari Krishna, Jpn. J. Appl. Phys. 33, 1305 (1994). doi:10.1143/JJAP.33.1305

13. P. Nithiananthi and K. Jayakumar, Sol. Stat. Commun. 137, 427 (2005). doi:10.1016/j.ssc.2005.12.025

14. C. Kittel, Introduction to Solid State Physics, 7th edition (Wiley Eastern Publications, 1998).

15. Zhigang Xiao, J. Appl. Phys. 86, 4509 (1999). doi:10.1063/1.371394

16. C. A. Duque, N. Porras-Montenegro, M. de Dios-Leyva, and L. E. Oliveira, J. Appl. Phys. 101, 093716 (2007). doi: $10.1063 / 1.2734097$

17. A. John Peter, Physica E 31, 125 (2006). doi:10.1016/j.physe.2005.10.010 